\title{
Pseudotumor inflamatorio por Fasciola hepatica: a propósito de un caso
}

\author{
Lucía Tume-Jara, Cindy Ugarte-Salvador, Javier Díaz-Ferrer y Alejandro Piscoya
}

\section{Inflammatory pseudotumor by Fasciola hepatica: a case report}

Introduction: Fasciola hepatica is a parasite of the class Trematoda. It commonly has been found in developing countries. When it infects humans is characterized by a triad of fever, pain in right upper quadrant and peripheral eosinophilia. We present a 67-year-old female from a rural town of the north of Lima, Peru, it was found abdominal pain, eosinophilia and focal hepatic lesions. For this reason, a hepatic mass was the initial suspicion. The hepatic biopsy was performed and one of the findings was eosinophilia. Fasciola hepatica infection should be considered as part of differential diagnosis in hepatic tumors with eosinophilia when the origin of the patient is from endemic areas of F. hepatica.

Key words: Fascioliasis, hepatic mass, eosinophilia.

Palabras clave: Fascioliasis, masa hepática, eosinofilia.
Escuela de Medicina, Universidad Peruana de Ciencias Aplicadas. Lima, Perú (LTJ, CUS, JP). Hospital Edgardo Rebagliati Martins. Lima, Perú (JD).

Los autores declaran que no presentan conflicto de interés. Los autores declaran que no presentan fuente de financiamiento.

Recibido: 20 de mayo de 2015 Aceptado: 19 de octubre de 2015

Correspondencia a: Lucía Tume-Jara luchia.201290@gmail.com

\section{Introducción}

$F$ Tasciola hepatica es un parásito de la clase Trematoda, común en países en desarrollo ${ }^{1,2}$. El ciclo biológico se inicia con la liberación de los huevos inmaduros en conductos biliares y luego en las heces del ganado. El desarrollo embrionario ocurre entre dos y 23 semanas, entre 10 y $30^{\circ} \mathrm{C}$, para después liberar los miracidios. Estos invaden a los caracoles de especie Lymnaea $y$ en estos tiene tres estadios: esporocisto, redia y cercaria. Las cercarias se liberan de los caracoles y forman quistes en vegetaciones acuáticas conocidos como metacercarias que cuando son ingeridas por mamíferos o humanos ocasionan la infección ${ }^{3}$.

En el mundo se han reportado casos de infección por $F$. hepatica con alta prevalencia en países de la región andina de Sudamérica, como Bolivia (65-92\%), Ecuador (24-53\%) y Perú (hasta $72 \%)^{4,5}$. Asimismo, la Organización Mundial de la Salud (OMS) ha reportado que siete millones de personas están en riesgo de contraer la infección en Perú 5 . En este país, las áreas endémicas son Puno (15,6-31\%), Junín (10,7-34,2\%) y Lima $(15 \%)^{4}$. La infección por $F$. hepatica en el ser humano se caracteriza por una triada compuesta por fiebre, dolor abdominal en el cuadrante superior derecho y eosinofilia ${ }^{2}$. Presentamos el caso clínico de una paciente con una fascioliasis que se presentó con dolor abdominal, eosinofilia y lesiones hepáticas hipodensas en la tomografía axial computarizada (TAC) abdominal $^{6}$.

\section{Caso clínico}

Mujer de 67 años, natural de Huaral y procedente de Lima Metropolitana, de ocupación dueña de casa, que consultó por un cuadro de seis meses de evolución de sensación de pesadez y distensión abdominal post-prandial con todo tipo de comida. En los últimos tres meses, estos episodios se asociaron a epigastralgia urente leve. Al examen físico destacaba dolor a la palpación profunda en el epigastrio, sin signos peritoneales.

Los exámenes de laboratorio demostraron una leucocitosis de $11.700 / \mathrm{mm}^{3}$ y una eosinofilia de $6.300 / \mathrm{mm}^{3}$, las fosfatasas alcalinas, GGT, tiempo de protrombina y alfa-fetoproteínas se encontraban dentro de límites normales. La serología para hepatitis $\mathrm{B}$ y $\mathrm{C}$ fue negativa.

En una ecografía abdominal, se encontraron dos imágenes quísticas en el hígado, una de $11 \mathrm{~mm}$ en el segmento VII y la otra de $45 \mathrm{~mm}$ en el segmento II.

Se realizó una TAC de abdomen que evidenció una imagen hipodensa de bordes irregulares, de 35 por 15 $\mathrm{mm}$ en el segmento hepático II (Figura 1). Se internó con el diagnóstico de un tumor hepático para realizarse una biopsia percutánea guiada por ecografía. Los hallazgos histopatológicos de la biopsia hepática fueron: un infiltrado inflamatorio compuesto por linfocitos, células plasmáticas y eosinófilos, estos últimos en gran cantidad. Se observaron además hepatocitos reactivos, regenerativos en el parénquima hepático (Figura 2 y 3 ). Por este motivo, se solicitó Fas2-Elisa para $F$. hepatica, el que tuvo un valor de 0,37 (valor normal: $<0,2$ ), muy sugerente de infección.

La paciente recibió tratamiento con triclabendazol 
Figura 1. Tomografía computarizada abdominal muestra una imagen hipodensa de bordes irregulares, de $35 \times 15$ mm en el segmento hepático II.

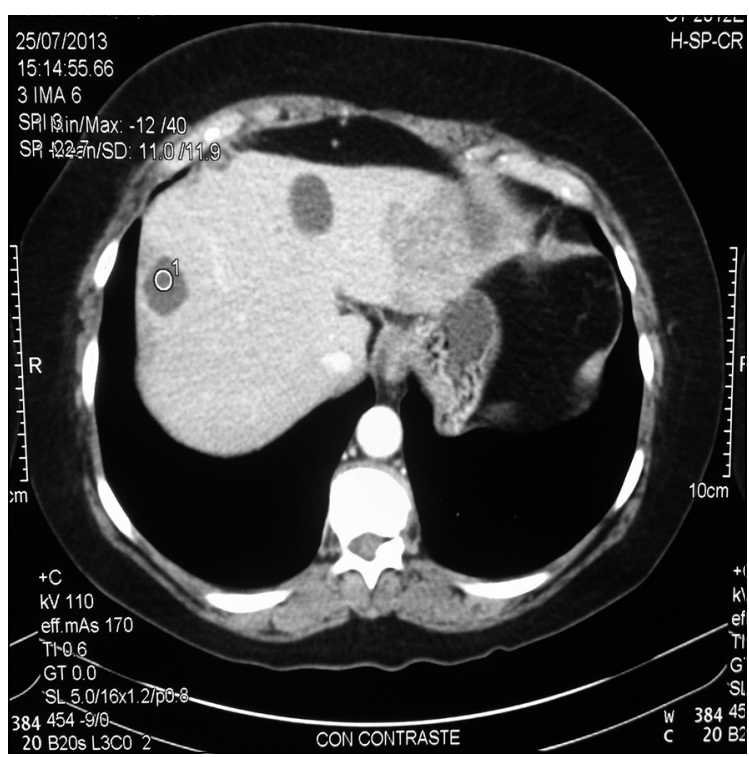

$10 \mathrm{mg} / \mathrm{kg}$, en dosis única. Evolucionó favorablemente; luego de dos meses desapareció la sintomatología y la eosinofilia. En la TAC abdominal realizada al sexto mes post-tratamiento, se evidenció regresión completa de la lesión hepática.

\section{Discusión}

Las infecciones del hígado por tremátodos, como el caso de $F$. hepatica, tienen como principal fuente de infección el consumo de alimentos o aguas contaminadas por metacercarias que se adhieren a las plantas acuáticas, como los berros y lechugas ${ }^{3,7}$. El cuadro clínico se divide en una fase aguda y crónica. La primera tiene una duración entre tres y cinco meses y los síntomas principales son fiebre prolongada y dolor abdominal; en el laboratorio generalmente se encuentra eosinofilia ${ }^{2}$. En la TAC de abdomen se pueden encontrar múltiples lesiones hipodensas, las cuales son semejantes a imágenes metastásicas. Mientras que, la fase crónica suele presentarse a partir de los seis meses; ésta en la mayoría de los casos es asintomática. Si hubiera síntomas se presentaría como un síndrome biliar obstructivo caracterizado por ictericia, fiebre y dolor abdominal ${ }^{2}$.

En este caso se sospechó en primer lugar un tumor hepático debido a la presencia en una TAC de una imagen hipodensa de bordes irregulares en el segmento hepático II. Por ello, se realizó una biopsia percutánea guiada por ecografía, cuyo hallazgo más relevante fue la presencia de un infiltrado inflamatorio con gran eosinofilia. Esto permitió el descarte de diagnósticos diferenciales de una masa hepática sólida como: hemangioma cavernoso, hiperplasia nodular focal, adenoma hepático, traumatismo, absceso hepático y hepatocarcinoma ${ }^{8}$.

El hemangioma cavernoso es la lesión hepática benigna

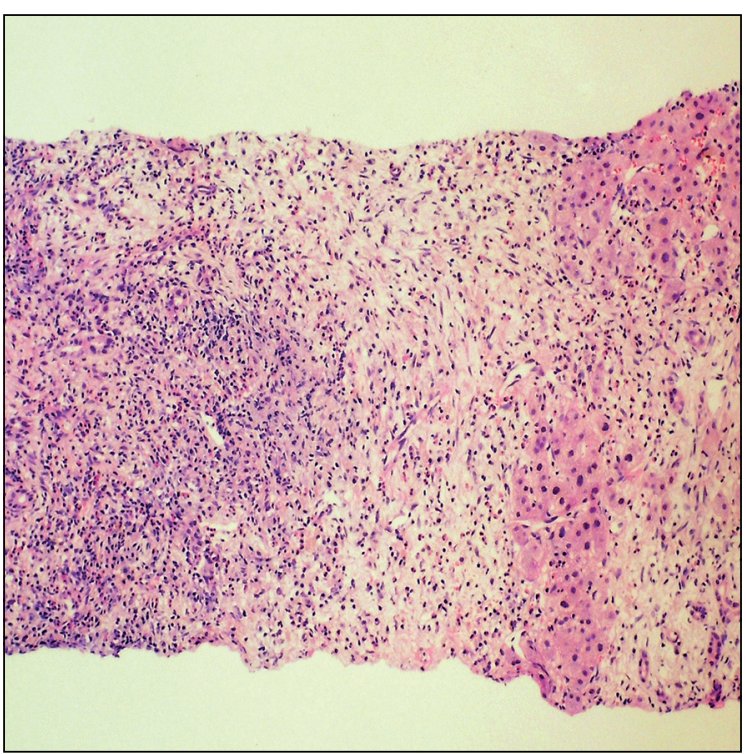

Figura 2. H-E.10x. Biopsia hepática con distorsión de su arquitectura por infiltrado inflamatorio crónico y presencia de abundantes eosinófilos con ensanchamiento de los espacios porta.

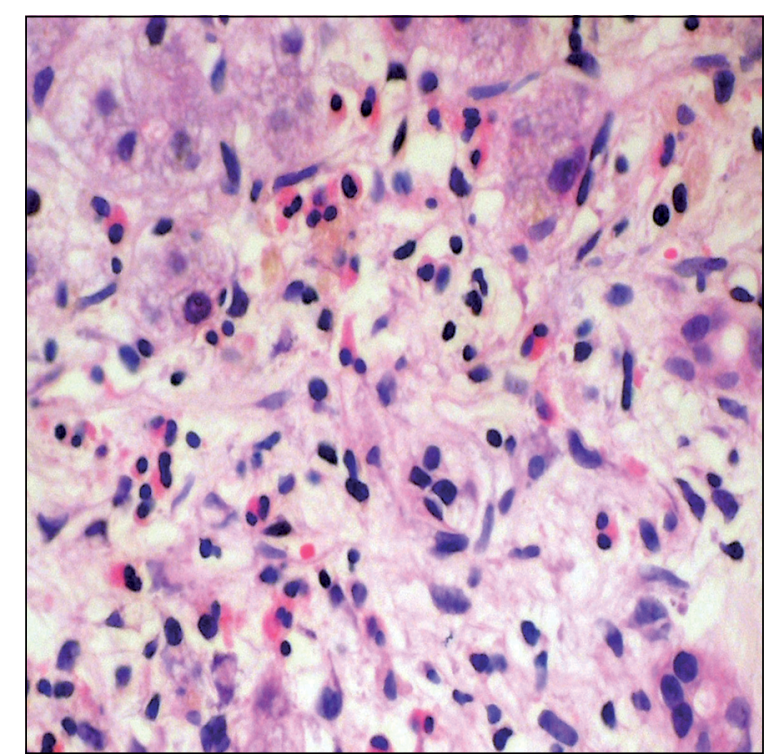

Figura 3. H-E.40x. Presencia de infiltrado inflamatorio linfoplasmocitario con abundantes eosinófilos y hepatocitos con cambios reactivos.

más común, predominante en mujeres y que cursan en forma asintomática. En este caso se descartó esta posibilidad por la presencia de eosinofilia en sangre y tejido hepático. La hiperplasia nodular focal, es el segundo tumor hepático más benigno, en mujeres entre la tercera y quinta década de la vida. El adenoma hepático se desestimó, ya que la paciente no se encontraba en edad fértil y no usaba anticonceptivos. Tampoco había antecedentes de algún traumatismo abdominal. El hepatocarcinoma se descartó 
debido a que no se encontraron hallazgos histológicos sugerentes como: nódulos displásicos con aumento de la vascularización arterial o pérdida de la fuente de sangre venosa portal, entre otros ${ }^{8}$. Por último, el cuadro clínico y la serología para hepatitis $\mathrm{B}$ y $\mathrm{C}$ fueron negativos.

Se han reportado casos similares de tumores hepáticos que tuvieron como diagnóstico final una infección por F. hepatica en países como Perú ${ }^{9}$, Chile $^{10}$, Turquía ${ }^{1,2,11,12}$ y Japón ${ }^{13}$. Sin embargo, aún existen dificultades para el diagnóstico de la fascioliasis hepática.

El método más utilizado para realizar el diagnóstico es el examen coproparasitológico que permite detectar los huevos de F. hepatica. Sin embargo, no es de utilidad en la fase aguda ${ }^{7}$. Por lo tanto, son necesarias las pruebas serológicas como FAS2-ELISA, hemaglutinación indirecta, fijación del complemento e inmunofluorescencia indirecta (IFI). FAS2-ELISA es una prueba serológica que detecta anticuerpos del tipo inmunoglobulina $\mathrm{G}$ del suero contra el antígeno FAS2 del parásito adulto ${ }^{14}$. Esta prueba es más rápida, fiable y tiene una sensibilidad de $95 \%{ }^{2}$. Otra técnica de alto rendimiento en la fase crónica es la detección de antígenos en deposiciones ${ }^{15}$.

De igual manera, se pueden usar imágenes como técnicas de diagnóstico. Un hallazgo sugerente de fascioliasis hepática son lesiones de tipo nodular o tubulares hipodensas que varían desde 20 a $30 \mathrm{~mm}$ de diámetro; éstas sobretodo se encuentran en el área subcapsular hepática ${ }^{11,13,16}$.

El fármaco de elección para la infección por $F$. hepatica es triclabendazol a una dosis de $10 \mathrm{mg} / \mathrm{kg}$, por uno o dos días; en general, es bien tolerado ${ }^{12,17}$. Este fármaco en
Perú es de uso veterinario. La paciente tuvo una buena respuesta clínica a este tratamiento.

\section{Conclusión}

En pacientes con dolor abdominal, eosinofilia y lesiones hepáticas focales se debe incluir en el diagnóstico diferencial a las infecciones parasitarias como F. hepatica; sobre todo en personas que proceden de países endémicos como Perú.

Agradecimientos. Agradecemos al Dr. Félix M. Querevalú Eche por analizar, interpretar y proveer las imágenes patológicas y al Dr. Germán F. Alvarado quien leyó y corrigió el manuscrito.

\section{Resumen}

Fasciola hepatica es un parásito de la clase Trematoda común en países en desarrollo. La infección en el ser humano se caracteriza por la triada de fiebre, dolor abdominal en el cuadrante superior derecho y eosinofilia. Se presenta el caso de una mujer de 67 años procedente de una zona rural al norte de Lima, con historia de dolor abdominal de seis meses de evolución, con una imagen hipodensa hepática en el TAC abdominal y eosinofilia. La biopsia hepática mostró un infiltrado inflamatorio con eosinofilia. En el diagnóstico diferencial en pacientes con un tumor hepático y eosinofilia, se deben incluir infecciones parasitarias como $F$. hepatica; sobre todo en pacientes que proceden de áreas endémicas.

\section{Referencias bibliográficas}

1.- Marcos L A, Terashima A, Gotuzzo E. Update on hepatobiliary flukes: fascioliasis, opisthorchiasis and clonorchiasis. Curr Opin Infect Dis 2008; 21: 523-30.

2.- Yilmaz B, Köklü S, Gedikoglu G. Hepatic mass caused by Fasciola hepatica: a tricky differential diagnosis. Am J Trop Med Hyg 2013; 89: 1212-3.

3.- Muro A, Pérez del Villar L, Velasco V, Pérez-Arellano J L. Infecciones por trematodos. Medicine 2010; 10: 3717-28.

4.- Loja Oropeza D, Alvizuri Escobedo J, Vilca Vásquez M, Avilés Gonzaga R, Sánchez Mercado M. Hematoma hepático subcapsular por fascioliasis. Rev Gastroenterol Perú 2003; 23: 142-8.

5.- Marcos L A, Tagle M, Terashima A, Bussalleu A, Ramírez C, Carrasco C, et al. Natural history, clinicoradiologic correlates, and response to triclabendazole in acute massive fascioliasis. Am J Trop Med Hyg 2008; 78 : 222-7.

6.- Fica A, Dabanch J, Farías C, Castro M, Jercic M I, Weitzel T. Acute fascioliasis- clinical and epidemiological features of four patients in Chile. Clin Microbiol Infect 2012; 18: 91-6.

7.- Espinoza J R, Terashima A, Herrera-Velit P, Marcos L A. Fasciolosis humana y animal en el Perú: impacto en la economía de las zonas endémicas. Rev Peru Med Exp Salud Publica. 2010; 27: 604-12.

8.- Venkatesh S K, Chandan V, Roberts L R. Liver masses: a clinical, radiologic, and pathologic perspective. Clin Gastroenterol Hepatol 2014; 12: 1414-29.

9.- Castillo Contreras O B, Frisancho Velarde O. Pseudotumor hepático en fasciolosis aguda. Acta Gastroenterol Latinoam 2013; 43: 53-8.

10.- Sapunar J, Braghetto I, Díaz J C, Brahm J, Apt W, Carreño L, et al. Fascioliasis hepática que simularon tumores hepáticos. Bol Chil Parasitol 2001; 57 (3-4): 59-65.

11.- Aksoy D Y, Kerimoglu U, Oto A, Ergüven S, Arslan S, Unal S, et al. Fasciola hepatica infection: clinical and computerized tomographic findings of ten patients. Turk J Gastroenterol 2006; 17: 40-5.

12.- Arslan F, Batirel A, Samasti M, Tabak F, Mert A, Özer S. Fascioliasis: 3 cases with three different clinical presentations.

Turk J Gastroenterol. 2012; 23: 267-71.

13.- Maeda T, Yamada H, Akao N, Iga M, Endo T, Koibuchi T, et al. Unusual radiological findings of Fasciola hepatica infection with huge cystic and multilocular lesions. Intern Med 2008; 47 : 449-52.

14.- Espinoza J R, Maco V, Marcos L, Sáez S, Neyra V, Terashima A, et al. Evaluation of Fas2-ELISA for the serological detection of Fasciola hepatica infection in humans. Am J Trop Med Hyg 2007; 76: 977-82.

15.- Espino A M, Borges A, Dumenigo B E. Coproantigenos de Fasciola hepatica de posible utilidad en el diagnóstico de la fascioliasis. Rev Panam Salud Publica 2000; 7: 225-31.

16.- Cevikol C, Karaali K, Senol U, Kabaalioğlu A, Apaydin A, Saba R, et al. Human fascioliasis: MR imaging findings of hepatic lesions. Eur Radiol 2003; 13: 141-8.

17.- Kaya M, Beştaş R, Çetin S. Clinical presentation and management of Fasciola hepatica infection: single-center experience. World J Gastroenterol 2011; 17 : 4899-904. 